\title{
Bilateral Thalamic Infarction After Traumatic Vertebral Artery Dissection
}

\author{
So Lyung Jung, Hyun Seok Choi, Jinhee Jang, Jaseong Koo, Yong Sam Shin, Kook-Jin Ahn, \\ Bum-soo Kim
}

Keywords: dissection, magnetic resonance imaging, stroke

doi:10.1017/cjn.2015.1

Can J Neurol Sci. 2015; 42: 208-209

\section{Case Presentation}

A 29-year-old female patient visited the emergency room for whirling dizziness and hypersomnolence. She had had a cervical chiropractic manipulation two weeks before admission. She had no previous history of stroke or vasculitis. Initial diffusionweighted images showed diffusion restriction in the bilateral thalami, occipital lobes, and brain stem. Subsequent magnetic resonance angiography (MRA) and source images of time of flight (TOF) MRA demonstrated luminal irregularities of the bilateral vertebral arteries and intramural hematoma on the wall of right vertebral artery. Catheter angiography showed an artery of Percheron. This case acknowledges that anatomic variation and a patient's history leads to the correct diagnosis.

\section{Discussion}

The thalamus is supplied by multiple perforators originating from the posterior communicating artery and the P1 and P2 segments of posterior cerebral arteries. An anatomic variation of the single trunk of the medial thalamic perforator, which branches out to bilateral smaller perforators, is called the artery of Percheron. The incidence of an artery of Percheron infarct is rare and is estimated to account for 0.1 to $0.3 \%$ of all ischemic strokes. ${ }^{1}$ Vertebral artery dissection is one of the causes for the artery of Percheron in posterior circulation infarction and its incidence is estimated to be two per 100,000 person-years. $^{2}$ An epidemiologic association between chiropractics and vertebral artery dissection has been reported in a German nationwide survey. ${ }^{3}$ Clinical symptoms were noticed
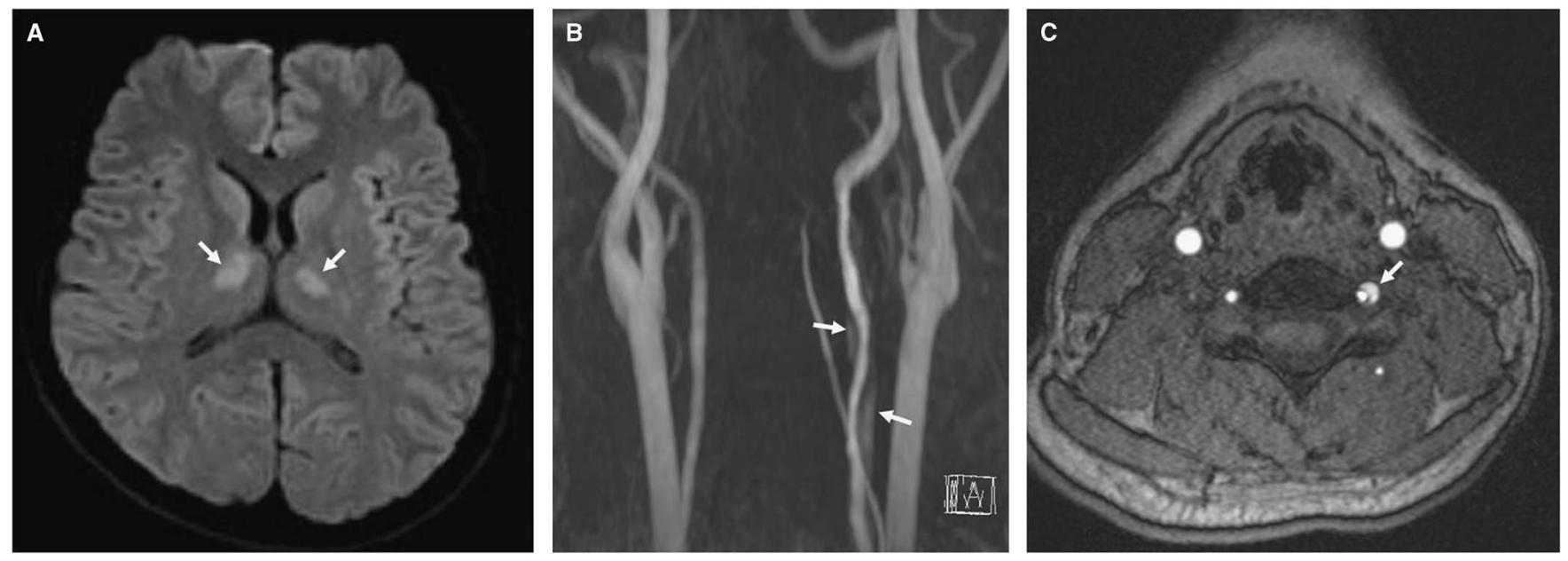

Figure 1: (A) High signal intensities (arrows) in bilateral thalami on diffusion-weighted image, suggestive of an acute infarct. (B) Irregular narrowing of the left vertebral artery and periluminal high signal intensities (arrows) on TOF-MRA. (C) A crescent-shaped high signal at the vertebral arterial wall on a TOF source image, suggestive of a dissection.

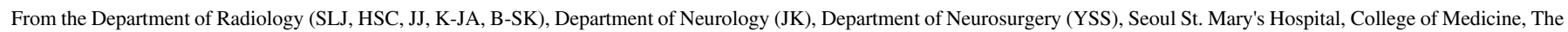
Catholic University of Korea.

Received June 3, 2014. Final Revisions Submitted December 4, 2014.

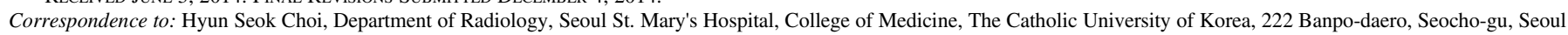
137-701, Republic of Korea. Email: hschoi@catholic.ac.kr. 


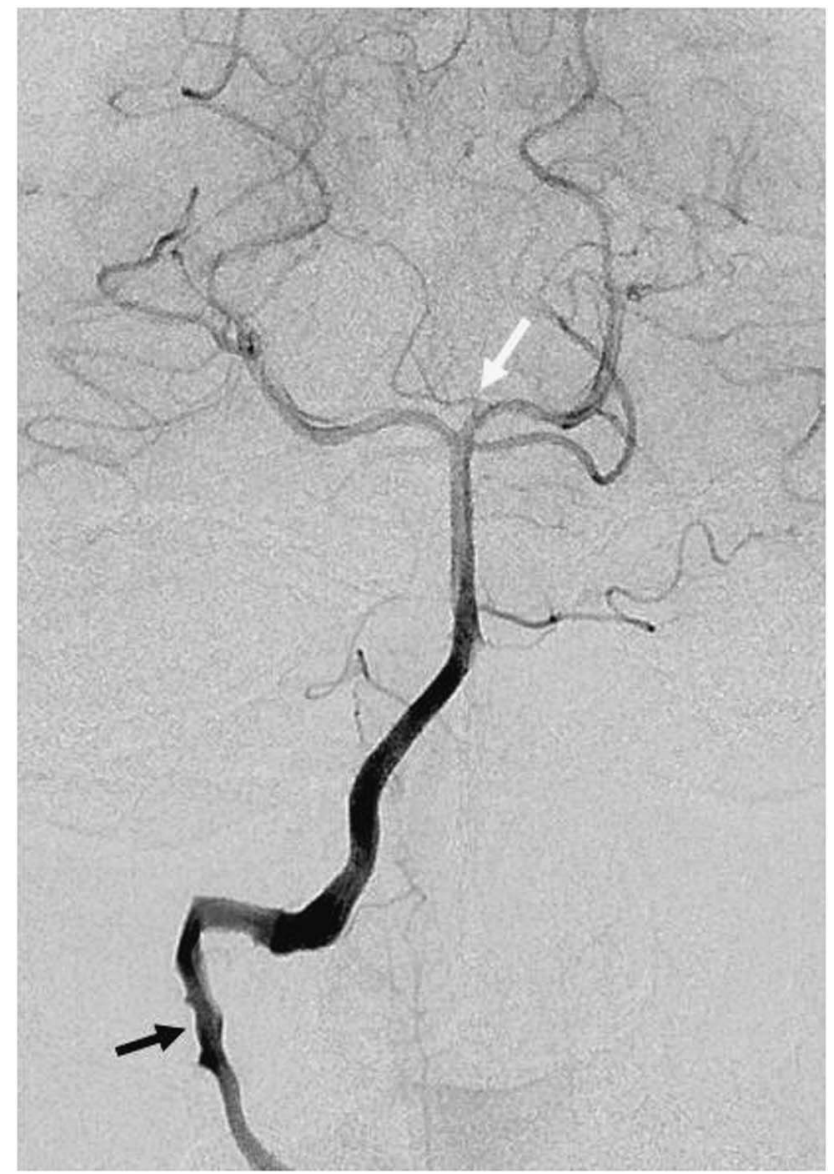

Figure 2: Catheter angiography with a right vertebral arterial injection shows an irregular luminal narrowing of right vertebral artery, suggestive of a dissection (black arrow). A single trunk of the bilateral thalamic perforator, artery of Percheron, originates from the left posterior cerebral artery (white arrow). within 12 hours after neck manipulation in $55 \%$ of patients. The prognosis was relatively benign in 34 of 36 patients of vertebral artery dissection with prior chiropractic neck manipulation. However, one patient died and the others remained in a persistent vegetative state. ${ }^{3}$

\section{Disclosures}

Authors have nothing to disclose.

\section{REFERENCES}

1. Lazzaro NA, Wright B, Castillo M, et al. Artery of percheron infarction: imaging patterns and clinical spectrum. AJNR. 2010;31:1283-9.

2. Preul C, Joachimski F, Witte OW, Isenmann S. Bilateral vertebral artery dissection after chiropractic maneuver. Clin Neuroradiol. 2010;20:255-9.

3. Reuter U, Hamling M, Kavuk I, Einhaupl KM, Schielke E. Vertebral artery dissections after chiropractic neck manipulation in Germany over three years. J Neurol. 2006;253:724-30. 